\title{
Acute Pregabalin Withdrawal: A Case Report and Review of the Literature
}

\author{
Jaclyn A. Barrett, PharmD \\ Lindsay M. Kittler, PharmD, BCPS \\ Clement Singarajah, MD, FCCP \\ Phoenix VA Medical Center \\ Phoenix, AZ 85012
}

\begin{abstract}
Objective: Pregabalin is a commonly prescribed GABA analog most commonly used for the treatment of neuralgia. Recently, case reports on pregabalin have been published describing episodes that may be associated with withdrawal-like symptoms after extended or aggressive therapy. This report describes a case in which long term exposure of high dose pregabalin may have resulted in acute withdrawal, and outlines the subsequent medical management of these symptoms.

Case Summary: A 61-year-old male presenting with severe agitation presumed to be withdrawal from long term and high dose exposure to pregabalin. Medical management included the use of haloperidol, diphenhydramine, lorazepam and the addition of clonidine over the course of several days for the pharmacological management of withdrawal symptoms.
\end{abstract}

Discussion: Although case reports are available to guide clinicians in the recognition of acute pregabalin withdrawal, definitive evidence on how best to treat these patients remains severely limited. With an increase in the prescribing practices of pregabalin, insight into the acute management by fellow clinicians is further needed.

Conclusion: Caution must be practiced when prescribing and educating patients on the use of pregabalin to prevent associated withdrawal-like symptoms. In addition, documentation by the medical community on methods utilized to treat pregabalin withdrawal syndromes remains crucial for the advancement of patient care. Benzodiazepines and clonidine are the current therapies that have been documented as potentially effective treatment modalities at this time.

\section{Introduction}

Pregabalin is a gamma-aminobutyric acid (GABA) analogue currently FDA labeled for diabetic peripheral neuropathy, fibromyalgia, neuropathic pain, partial seizures and postherpetic neuralgia, and is used off-label in various psychiatric disorders. It is proposed that pregabalin binds strongly to an alpha-2-delta subunit on voltage gated calcium channels within the central nervous system. Pro-nociceptive neurotransmitter release that is dependent upon calcium is thereby reduced. Additional mechanisms for pregabalin's efficacy may be comprised of noradrenergic and serotonergic pathways involved in pain transmission (1). 


\section{Case Presentation}

We report the case of a 61-year-old man who presented with sudden and severe withdrawal like symptoms characterized by family as extreme agitation with combative behavior, diaphoresis, tachycardia, hypertension, tremors, and incontinent diarrhea. Reportedly, prior to admission, the patient had become increasingly somnolent with multiple falls and psychotic behavior similar to one responding to internal stimuli. A review of his home prescription vials revealed a presumed, excessive consumption of immediate-release oxycodone and pregabalin over the last few days and up to a week. His past medical history included chronic obstructive pulmonary disease, osteoarthritis, neuropathic pain, hypothyroidism, hyperlipidemia, anxiety and major depressive disorder. A thyroid stimulating hormone level was checked upon admission and was within normal limits with no other metabolic explanation for his symptoms identified. The patient did present with a prior medical history of opioid abuse with previous enrollments in substance abuse programs, but compliance with his current pain management contract was confirmed using the state's controlled substance prescription monitoring program database.

Upon admission through the emergency room, the patient remained severely agitated with increasingly combative behavior despite the administration of the antipsychotic, haloperidol. Without relief, the benzodiazepine, lorazepam, was administered resulting in a paradoxical effect causing increased aggression and agitation and thus discontinued. Implementation of scheduled hydromorphone was administered for acute opioid withdrawal but similarly provided minimal symptom abatement yet, maintained adequate analgesia. After medication review and reconciliation, the patient was currently on a prescribed, scheduled dose of pregabalin $300 \mathrm{mg}$ twice daily over the course of approximately eight months for the treatment of neuropathic pain. Although initially concerned for potential overdose, all symptoms upon presentation were deemed to be consistent with a withdrawal syndrome, pregabalin being the most likely culprit given lack of symptom abatement with the use of benzodiazepines and opioids with no other likely cause upon review of his home medications and laboratory data.

After transferring to the Intensive Care Unit, the patient was further treated with intravenous haloperidol $10 \mathrm{mg}$ and diphenhydramine $50 \mathrm{mg}$ every 4 hours providing only minimal relief while protecting both the safety of the patient and staff. Management of acute pregabalin withdrawal was further complicated by the inability to provide pregabalin orally, due to disorientation and the physical inability to maintain placement of a nasogastric tube. Sedation and mechanical ventilation was not an option as the patient's established wishes were a Do Not Intubate (DNI) status. Sublingual clonidine $0.1 \mathrm{mg}$ was later administered every four hours and up to every hour if needed, in addition to the haloperidol and diphenhydramine. The anti-adrenergic agent clonidine was most effective in providing withdrawal symptom reduction, and the haloperidol and diphenhydramine were rapidly tapered off. In addition, our patient developed urinary retention necessitating the discontinuation of diphenhydramine. The discontinuation of diphenhydramine lead to a retrial of lorazepam which provided effective relief for the management of his later course agitation with no paradoxical agitation observed. 
This case demonstrates an issue of common occurrence, where a patient is withdrawing clinically from a drug with often non-specific symptom manifestations. Further confounding this clinical presentation, several possible sources of drug withdrawal required the medical team to address each drug individually in order to identify the offending agent. With this in mind, withdrawal from pregabalin, although not often considered a drug of abuse or associated with intense withdrawal, may only be identified by ruling out other suspected agents, such as in this patient scenario.

\section{Discussion}

In review of current literature, case reports have arisen that describe symptoms of withdrawal from abrupt discontinuation of pregabalin. Several case reports describe a withdrawal-like syndrome to include signs and symptoms such as agitation, anxiety, confusion, gastrointestinal distress, tachycardia and palpitations, similar to benzodiazepine withdrawal (2-4).

Oaklander and Buchbinder (2) report a case involving an 80-year-old woman prescribed pregabalin $125 \mathrm{mg}$ three times daily for 49 weeks as treatment for postherpetic neuralgia. Within 36 hours of abrupt discontinuation, the patient developed severe nausea, headache, imbalance, delirium and anorexia. Over the course of several weeks, the patient developed hemodynamic instability and continued to experience episodes of ataxia for a six month duration.

Another report by Karosin et al. (3), describes a 47-year-old male who reportedly consumed a total daily dose of $7,500 \mathrm{mg}$ of pregabalin daily in addition to alcohol and cocaine abuse. In a failed attempt to wean off pregabalin the patient developed what was described to be vegetative withdrawal symptoms, sweating, restlessness, hypertension, tremor and pregabalin cravings. Upon admission, the patient received treatment with benzodiazepines without relief and a slow taper of pregabalin was initiated yet, cravings continued which ultimately resulted in the patients continued abuse of this GABA analog.

In a case report by Norgaard et al. (4), a 38 year-old man was self-administering $8.4 \mathrm{~g}$ pregabalin daily and within 36 hours of cessation of pregabalin developed auditory hallucinations and suicidal ideation in addition to sweating, anxiety and tachycardia. Pregabalin was reinitiated at $600 \mathrm{mg}$ per day in addition to supplemental chlordiazepoxide and quetiapine. Acute symptoms resolved within 48 hours yet the symptoms of psychosis remained present until several weeks after discontinuation.

In addition, case reports regarding gabapentin withdrawal have been published to include similar clinical presentations. Gabapentin, another GABA analog, is FDA approved for the treatment epilepsy, neuralgia and restless leg syndrome, and is widely used in the prophylaxis of migraines, headaches and ethanol withdrawal (5). It is hypothesized that the mechanism of withdrawal is associated with increased activity of the enzyme responsible for producing GABA, similar to the mechanism behind ethanol and benzodiazepine withdrawal (6). 
Hellwig et al. (6) and Mah et al. (7), describe similar symptoms of irritability, confusion and agitation unrelieved by the administration of benzodiazepines. Gabapentin was identified as the causative agent of withdrawal after reinstatement of the GABA analog provided relief in both case reports. In addition to drug related factors, potential patient related risk factors that may increase the susceptibility of withdrawal include advanced age and history of psychiatric illness.

Currently, pregabalin is a schedule $\mathrm{V}$ controlled substance due to its potential for addiction and abuse (8). According to the manufacturer, there are no current recommendations for the management of an acute drug withdrawal syndrome from pregabalin, yet current practice suggests that a gradual taper, generally recommended with all extended controlled substance therapy, may be warranted. This report suggests that clonidine may be an effective agent in the management of acute agitation associated with pregabalin withdrawal. Literature has historically supported the use of clonidine in a variety of drug withdrawal syndromes owing to its known central alpha 2 agonist activity, but remains underutilized amongst providers in current practice (9-11). Another alternative to consider is dexmedetomidine, a centrally acting, alpha 2 adrenoceptor agonist which has demonstrated its efficacy as an adjunctive treatment for refractory alcohol withdrawal (12). Due to concomitant hypertension and institutional cost, dexmedetomidine was considered second line in the event that clonidine was ineffective for our patient.

Although case reports are available to guide clinicians in the recognition of acute pregabalin withdrawal, definitive evidence on how best to treat these patients remains severely limited. With an increase in the prescribing practices of pregabalin, insight into the acute management by fellow clinicians is further needed to provide evidence-based guidance in the management of these patients. As for our clinical case of acute pregabalin withdrawal in the presence of hallucinatory features, we advise against the use of benzodiazepines due to the risks of paradoxical agitation, and would suggest withholding their use until the latter stages of withdrawal. If possible, as was not in our case, initiating a pregabalin taper would ideally preferred. We also propose that the use of clonidine has clinically shown, as demonstrated in this patient case, to provide appropriate supportive treatment for the management of acute pregabalin withdrawal.

\section{References}

1. Pregabalin. DrugPoint Summary. Micromedex. Truven Health Analytics, Inc. Greenwood Village, CO. Available at: http://www.micromedexsolutions.com. Accessed April 26, 2014.

2. Oaklander AL, Buchbinder BR. Pregabalin-withdrawal encephalopathy and splenial edema: a link to high-altitude sickness? Ann Neurol. 2005;58(2):309-12. [CrossRef] [PubMed]

3. Karosin C, Kofler M, Mayr A, et al: Pregabalin: A treatment option for dystonia? Neurol Sci. 2012;33(2):351-4. [CrossRef] [PubMed]

4. Nordgaard J, Jurgens G. A case of pregabalin abuse. Clin Toxicol. 2013;51(4):320 
5. Gold Standard, Inc. Gabapentin. Clinical Pharmacology [database online]. Available at: http://www.clinicalpharmacology-

ip.com/Forms/drugoptions. aspx?cpnum=271\&n=Gabapentin\&t=0 Accessed: 5/19/2015.

6. Hellwig TR, Hammerquist R, Termaat J. Withdrawal symptoms after gabapentin discontinuation. Am J Health Syst Pharm. 2010;67:910-2. [CrossRef] [PubMed]

7. Mah L, Hart M. Gabapentin withdrawal: case report in an older adult and review of the literature. J Am Geriatr Soc. 2013;61(9):1635-7. [CrossRef] [PubMed]

8. LYRICA $®$ (pregabalin) CV Safety Info. Available at: http://www.lyrica.com. Accessed April 27, 2014.

9. Baumgartner GR, Rowen RC. Clonidine vs chlordiazepoxide in the management of acute alcohol withdrawal syndrome. Arch Intern Med. 1987;147:1223-6. [CrossRef] [PubMed]

10. Baumgartner GR, Rown RC. Transdermal clonidine versus chlordiazepoxide in alcohol withdrawal: a randomized, controlled clinical trial. South Med J. 1991;84:31221. [CrossRef] [PubMed]

11. Robinson BJ, Robinson GM, Mailing TJB, Johnson RH. Is clonidine useful in the treatment of alcohol withdrawal? Alcohol Clin Exp Res. 1989;13:95-8. [CrossRef] [PubMed]

12. Tolonen J, Rossinen J, Alho H, Harjola VP. Dexmedetomidine in addition to benzodiazepine-based sedation in patients with alcohol withdrawal delirium. Eur J Emerg Med. 2013;20:425. [CrossRef] [PubMed] 